\title{
La pharmacoépidémiologie : bases réglementaires, approches méthodologiques et champ d'application
}

S. Ahid, ${ }^{1}$ K. Belaguide et Y. Cherrah ${ }^{1}$

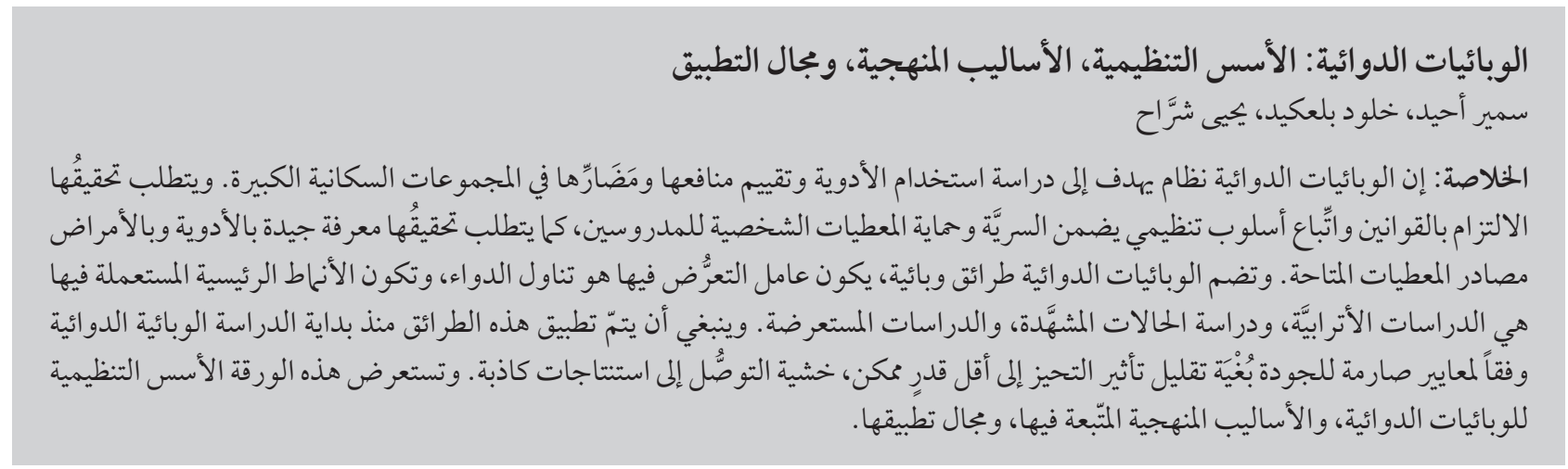

RÉSUMÉ La pharmacoépidémiologie est une discipline visant à étudier l'utilisation des médicaments et l'évaluation de leurs effets bénéfiques ou indésirables sur de grandes populations. Sa réalisation requiert le respect de lois et le suivi d'une démarche réglementaire permettant d'assurer la confidentialité et la protection des données personnelles issues de ces études; elle nécessite de disposer d'informations solides sur les médicaments et les pathologies en ayant recours aux différentes sources de données disponibles. La pharmacoépidémiologie reprend les méthodes propres à l'épidémiologie où le facteur d'exposition est constitué par la prise du médicament, et dont les principaux types utilisés sont les études de cohorte, études cas-témoins et études transversales. L'application de ces méthodes doit se faire selon des critères de qualité prévus dès la conception d'une étude pharmacoépidémiologique afin de minimiser l'effet des biais amenant à des conclusions erronées.

\section{Pharmacoepidemiology: regulatory basis, methodological approaches and scope}

ABSTRACT Pharmacoepidemiology is a discipline that studies the use of drugs and evaluation of their beneficial or adverse effects on large populations. It requires compliance with laws and maintaining a regulatory approach in order to ensure confidentiality and protection of personal data. It also requires good knowledge of drugs and diseases and the use of the different available data sources. Pharmacoepidemiology incorporates epidemiological methods (cohort, case-control and cross-sectional studies) where the exposure is drug intake. These methods must be applied at the conception of the pharmacoepidemiological study in order to minimize the effect of bias hich can lead to false conclusions. This paper reviews the regulatory basis, methodological approaches and scope of pharmacoepidemiology.

'Équipe de Pharmacoépidémiologie \& Pharmacoéconomie, Laboratoire de Pharmacologie-Toxicologie, Faculté de Médecine et de Pharmacie, Rabat (Maroc) (Correspondance à adresser à S. Ahid : samir.ahid@um5s.net.ma).

Reçu : 10/06/10; accepté : 22/06/10 


\section{Introduction}

L'arrivée d'un nouveau médicament s'accompagne de la publication et de la communication des résultats d'essais contrôlés démontrant l'efficacité et la tolérance du produit. Les conclusions que l'on peut tirer de ces essais sont régulièrement et quasi inévitablement limitées par certaines caractéristiques de ces études : par exemple, leur courte durée et le suivi d'un nombre limité de malades, ce qui ne permet pas de détecter les effets indésirables rares et ceux qui ne s'observent qu'en cas de traitement prolongé $[1,2]$.

Ainsi, il y a toujours lieu de discuter la transposabilité des résultats de tels essais à une situation clinique réelle et leur généralisation à l'ensemble de la population. Il est donc nécessaire de disposer d'éléments plus objectifs et plus convaincants, ce qui est le but principal de la pharmacoépidémiologie qui s'impose comme une science incontournable de l'évaluation du médicament avec des techniques en constante innovation en vue de produire des connaissances pour évaluer le bénéfice et le risque de l'exposition à un médicament en situation réelle.

La pharmacoépidémiologie peut être définie comme la discipline mettant en application les méthodes et/ou le raisonnement épidémiologique(s) pour évaluer, souvent sur de grandes populations, l'efficacité, le risque et l'usage des médicaments [3]. Cette science d'évaluation assez récente fait appel à des études observationnelles pour déterminer les effets favorables et défavorables des médicaments dans la pratique courante après leur autorisation de mise sur le marché (AMM) [4]

\section{Historique}

Si l'on attribue la naissance sémantique du terme «pharmacoépidémiologie » à un article de D.H. Lawson paru dans le British Medical Journal en 1984, il faut de garder à l'esprit que la nouveauté du terme ne recouvrait pas une nouveauté absolue dans l'action [5].

En effet, la nécessité des études pharmacoépidémiologiques est apparue au début des années 1960 lors de l'épidémie de malformations liée à la prise de thalidomide par des femmes enceintes $[4,6]$ et la survenue d'adénocarcinomes vulvaires chez des jeunes filles dont les mères avaient été traitées par le diéthylstilbestrol durant leur grossesse $[7,8]$. Puis il y eut l'épidémie de syndromes éosinophiliemyalgie liés à la consommation de tryptophane en 1989 [9] et les cas d'hypertension artérielle pulmonaire primitive associés à la prescription de dexfenfluramine comme anorexigène, ou encore la survenue d'atteintes valvulaires aux États-Unis causées par la fenfluramine en 1997 [10].

Deux ans plus tard, la cérivastatine utilisée en tant que hypolipémiant a été retirée du marché suite à l'apparition d'un risque de rhabdomyolyse [11]. On assista par la suite en 2004 au retrait du rofécoxib dont l'utilisation a été associée au risque de hausse d'infarctus du myocarde, les conflits d'intérêts ayant affecté les études de rofécoxib réalisées au début des années 2000 et avant le retrait du médicament par le laboratoire [12,13]. Il est probable que si ces études n'avaient pas entretenu le doute, cela aurait pu permettre un retrait anticipé, diminuant peut-être le nombre de victimes de ce médicament [14].

En novembre 2009, l'Agence française de sécurité sanitaire des produits de santé (Afssaps) suspend l'autorisation de mise sur le marché des médicaments contenant du benfluorex suite à une étude mettant en perspective son efficacité modeste dans la prise en charge du diabète de type 2 et confirmant le risque d'atteinte des valves cardiaques. Ce retrait a eu lieu avec un retard de dix ans au minimum selon l'Inspection générale des affaires sociales (IGAS) [15].
D'après ces exemples d'effets indésirables, on constate que depuis les années 1960, l'emploi thérapeutique de molécules a suscité l'intérêt des pharmacoépidémiologistes qui ont contribué par leurs études à l'évaluation du risque, de l'efficacité et de l'usage des médicaments. Les résultats de leurs études ont sans aucun doute aidé à promouvoir l'utilisation rationnelle des médicaments, qui mène à une meilleure qualité des soins de santé.

Notamment, les études réalisées suivent une méthodologie stricte; elles sont élaborées et effectuées suivant un protocole précis, avec l'accord d'une commission d'éthique, et publiées. Il est important de signaler que les conflits d'intérêts des firmes peuvent, dans la réalité, fortement influencer les choix et aboutir ainsi à une « information » qui est incomplète et trompeuse.

\section{Champ d'application}

La pharmacoépidémiologie a trois principaux aspects qui sont les suivants.

\section{La pharmacoépidémiologie comme outil sécuritaire}

La pharmacoépidémiologie intervient comme dispositif de détermination des effets favorables ou indésirables observés chez les populations réellement utilisatrices. Cette détermination s'effectue à travers des effets cliniquement pertinents (qualité de vie, morbidité, mortalité) en faisant appel à une population bien plus importante, non sélectionnée et incluant l'analyse de la coprescription, des cofacteurs et les impacts écologiques $[1,2,16]$.

\section{La pharmacoépidémiologie comme outil d'évaluation de la prescription et la consommation}

- Les études de prescriptions ont pour but la description (qualité, attitude) des acteurs de santé ou des utilisateurs d'un médicament et celle des conditions dans lesquelles ce 
médicament est utilisé (posologie, respect des contre-indications et des conditions d'arrêt du traitement, surveillance du traitement, modalités d'administration) [17].

- Les études de consommation visent l'obtention d'informations sur les quantités, les volumes d'utilisation et les profils des médicaments utilisés, ainsi que l'évaluation de la population effectivement traitée [17].

\section{La pharmacoépidémiologie comme outil d'évaluation des coûts}

La dimension économique est devenue indissociable de la médecine de soins. La pharmacoéconomie tient une place grandissante dans l'évaluation de l'ensemble des conséquences médicoéconomiques imputables à l'usage d'un médicament. Elle identifie, mesure et compare les coûts (ressources consommées) et les effets (bénéfices, avantages) $[18,19]$.

\section{Aspects réglementaires et législatifs}

Les études pharmacoépidémiologiques sont des études observationnelles qui n'engendrent pas de modification de la prise en charge médicale habituelle des personnes concernées et ne portent pas atteinte à l'intégrité physique ou psychique du patient. Par conséquent, ces études ne s'inscrivent pas dans le champ d'application des lois relatives à la protection des personnes qui se prêtent à des recherches biomédicales. En revanche, les études pharmacoépidémiologiques conduisent à la constitution de fichiers informatiques comportant des informations nominatives ou indirectement nominatives sur les personnes participant aux études.

Plusieurs dispositifs interviennent pour protéger la vie privée et la confidentialité des informations des personnes impliquées dans la recherche.
Ces dispositifs constituent un corps de règles résultant, outre de la loi, de règlements internes de sociétés savantes, de principes émanant d'instances représentatives et de règles de bonnes pratiques entre professionnels [20].

En France, au cours des deux dernières décennies, des lois ont été adoptées sur la protection des données qui régissent l'utilisation des renseignements personnels :

- La loi n 78-17 du 6 janvier 1978 relative à l'informatique, aux fichiers et aux libertés qui porte sur la collecte, l'enregistrement et la conservation des renseignements personnels [20]. Le contrôle de l'application de cette loi et de son respect est assuré par la Commission nationale de l'informatique et des libertés (CNIL) à laquelle tout protocole d'étude pharmacoépidémiologique traitant des données nominatives doit être soumis pour avis [21-23]. Lorsqu'il s'agit de données médicales, l'avis du Comité consultatif sur le traitement de l'information en matière de recherche dans le domaine de la santé (CCTIRS) est requis avant la soumission du dossier à la CNIL [24,25]. Après l'obtention des avis du CCTIRS et de la CNIL, l'étude pharmacoépidémiologique doit faire l'objet d'une soumission à la Commission ordinale de l'Ordre des médecins pour l'application de l'article L 4113-6 du Code de la Santé publique, du fait que les médecins observateurs seront rémunérés pour leur participation [26].

- La loi relative à l'informatique, aux fichiers et aux libertés a été modifiée par la loi no 2004-801 du 6 août 2004 relative à la protection des personnes physiques à l'égard des traitements de données à caractère personnel, dans le but de l'harmoniser avec le droit européen [20].

Aux États-Unis, il n'y a pas de loi d'ensemble (omnibus bill), régissant la protection des données. La tendance est plutôt d'élaborer des normes fédérales détaillées de protection de la vie privée applicables à divers secteurs de la société. En effet, en 1996, le Congrès américain a adopté une loi fédérale : il s'agit du Health Insurance Portability and Accountability Act (HIPAA) de 1996 qui prévient l'utilisation ou la divulgation, intentionnelle ou non intentionnelle, des renseignements personnels protégés sur la santé [27]. Cette loi fédérale a deux règlements, qui sont le Privacy Rule (règlement sur la confidentialité) et le Security Rule (règlement sur la sécurité des données).

Le Maroc dispose d'une loi nationale qui protège les données personnelles. Il s'agit de la loi nº 08-09 relative à la protection des personnes physiques à l'égard du traitement des données à caractère personnel, qu'on peut utiliser comme plateforme et cadre réglementaire régissant les études pharmacoépidémiologiques. Cette loi, promulguée par le Dahir n²1.09.15 en date du 18 février 2009, a été publiée au Bulletin officiel n'5711 du 23 février 2009 et suivie du décret $n^{\circ} 2$ 09-165, en date du 21 mai 2009, lequel a été publié au Bulletin officiel n 5744 du 18 juin 2009. Elle place le Maroc parmi les premiers pays arabes et africains disposant d'un système de protection aussi complet, et le positionne parmi les destinations sûres du point de vue de la circulation des données personnelles.

\section{Sources de données en pharmacoépidémiologie}

Les différentes sources de données permettent à la fois d'obtenir des informations plus ou moins détaillées sur l'exposition au médicament (nombre de boîtes prescrites, vendues, remboursées, données démographiques sur la population traitée) et sur les données de morbi-mortalité des pathologies d'intérêt avec lesquelles interfèrent ces médicaments [28,29]. On distingue principalement les différentes sources de données suivantes. 


\section{Littérature et Internet}

\section{Bases de données d'articles scientifiques}

Elles présentent des références d'articles de revues (dépouillement d'articles de périodiques), d'ouvrages publiés ou de littérature grise (documents non publiés ou à nombre restreint d'exemplaires) [30-35]. Il existe de nombreuses bases de données bibliographiques.

- Lesbasesdedonnéesbibliographiques internationales françaises, comme celles de l'Institut de l'information scientifique et technique, ou INIST (PASCAL, FRANCIS et articles@ INIST) la base documentaire de la banque de données en santé publique (BDSP).

- Les bases de données bibliographiques internationales étrangères, telles que PubMed (Medical litterature analysis and retrieval system online), The Cochrane Library et EMBASE. On cite aussi les bases BIOSIS previews, Current Contents, HealthSTAR, Web of Science.

\section{Sites des agences gouvernementales et des sociétés savantes}

Les sites des agences gouvernementales diffusent des informations sur les médicaments, notamment des études et des statistiques.

En France, on trouve le ministère du Travail, de l'Emploi et de la Santé, le Haut Conseil de la santé publique, la Caisse nationale de l'assurance maladie des travailleurs salariés (CNAMTS) et l'Institut national de la santé et de la recherche médicale (Inserm).

Il existe également plusieurs sociétés savantes selon la discipline dont elles relèvent: par exemple, American Society for Pharmacology and Experimental Therapeutics, la Société française de pharmacologie et de thérapeutique, la Société française de santé publique, la Société marocaine de pharmacologie, la Société marocaine de pharmacovigilance, etc.

\section{Bases de données}

L'une des principales évolutions méthodologiques dans le domaine de la pharmacoépidémiologie a été l'émergence de bases de données de taille et de qualité substantielles. La constitution de ces bases informatisées qui regroupent des informations cliniques ou administratives relevées de façon systématique a grandement facilité la réalisation des études observationnelles. Elles comportent des informations démographiques, les motifs des consultations, les diagnostics, les prescriptions, les motifs d'hospitalisations éventuelles [34]. On peut classer les bases de données en deux groupes : les bases de données médicales et administratives [36,37].

\section{Registres}

Un registre est défini comme un recueil continu et exhaustif de données nominatives intéressant un ou plusieurs événements de santé dans une population géographiquement définie. Il est constitué à des fins de recherche épidémiologique et de santé publique par une équipe ayant les compétences appropriées [38,39].

\section{Observatoires du médicament}

L'observatoire du médicament est un organisme étudiant les médicaments après leur mise sur le marché. Il n'est pas unique, mais regroupe un ensemble de structures publiques et privées pouvant travailler ensemble ou séparément. C'est un outil précieux de la pharmacoépidémiologie. Cet observatoire a pour mission d'observer et d'analyser les pratiques quotidiennes de prescription et de consommation des médicaments, d'étendre les connaissances acquises lors des phases pré-AMM, de contribuer à la validation de nouvelles thérapeutiques et donc à l'extension d'AMM de médicament en ayant déjà une, et de contribuer à l'évaluation médicoéconomique des produits de santé [38].

\section{Notifications de pharmacovigilance}

La notification spontanée des effets indésirables des médicaments par les professionnels de santé constitue la pierre angulaire de tout système de pharmacovigilance et représente une source importante d'informations en pharmacoépidémiologie. Elle permet la surveillance de tous les médicaments nouveaux et anciens. Cette surveillance s'opère à grande échelle puisque 70 pays disposent actuellement de centres nationaux de pharmacovigilance.

\section{Approches méthodologiques}

L'approche pharmacoépidémiologique est généralement observationnelle en ce sensqu'ellesefixe pour objectifde décrire la réalitételle qu'elle est, sansintervention sur le cours naturel des évènements. Les études épidémiologiques d'observation utilisées en pharmacoépidémiologie sont les suivantes.

\section{Les études transversales}

Les enquêtes transversales sont des études épidémiologiques, principalement descriptives, évaluatives et parfois analytiques. Elles permettent de mesurer la prévalence d'une variable (exposition, événement, maladie, etc.) dans une population à un instant donné. En pharmacoépidémiologie, les études transversales peuvent, par exemple, être utilisées pour mesurer la prévalence d'une maladie ou d'un événement dans une population et la prévalence d'une exposition comme la consommation d'un médicament [39-49].

\section{Les études de cohorte}

L'étude de cohorte a pour but de rechercher l'existence d'une relation entre la survenue d'un problème de santé dans une population et la présence d'un facteur suspecté de pouvoir influencer cette survenue [50]. 
Le terme de cohorte désigne un groupe de sujets sélectionnés en fonction d'une ou de plusieurs caractéristique(s) commune(s), et suivis dans le temps dans le but d'identifier, de décrire ou de quantifier un phénomène [3].

Selon le moment de l'inclusion des sujets au sein de la cohorte, on distingue les cohortes fixes, dites encore fermées, dans laquelle toutes les personnes de la cohorte sont incluses au début de l'étude, sur une courte période, sans inclusion nouvelle au-delà, et les cohortes dynamiques, dites encore ouvertes dans lesquelles l'inclusion des personnes se poursuit au fur et à mesure queles premières personnes sont suivies, le temps de participation de chacune au suivi étant variable [29,51-57]

\section{Les études cas-témoins}

Les études cas-témoins sont des études épidémiologiques étiologiques qui mettent en évidence de façon rétrospective une relation entre une exposition à divers facteurs de risque potentiels et un état de santé. Elles comparent cette mesure de l'exposition entre le groupe de personnes malades, atteintes du problème de santé étudié (les cas), et le groupe de personnes indemnes de ce problème de santé (les témoins) [57,58].

En pharmacoépidémiologie, les études cas-témoins sont notamment intéressantes pour étudier l'association entre la prise d'un médicament et un événement indésirable de faible probabilité de survenue et/ou d'apparition retardée $[59,60]$.

\section{Les études écologiques}

Une étude écologique est une étude ou analyse basée sur des données populationnelles globales (données agrégées), disponibles à l'échelon d'une région ou d'un pays [61]. Elles permettent de tester une hypothèse. Elles partent de données déjà colligées au niveau des populations et essaient de les mettre en relation [62-64]. Ces données recueillies portent sur la fréquence, au sein de groupes, d'un problème de santé d'une part, et de l'exposition à un facteur de risque d'autre part.

D'autres types d'études peuvent être rencontrées telles que les études cas-témoins intra-cohorte, les études cas-cohorte, les études cas croisé ou case-crossover et les études caspopulation [3,65-68].

\section{Notions de biais en pharmacoépidémiologie}

Un biais est une erreur de raisonnement ou de procédure amenant à une représentation faussée de la réalité. Il revêt un caractère systématique et altère l'estimation dans un sens donné [3]. Ces erreurs méthodologiques sont très graves ; elles compromettent la validité de l'enquête et empêchentl'interprétation juste des résultats. Il faut les distinguer des erreurs aléatoires qui n'entrainent qu'un manque de précision sur le paramètre estimé [40]. En présence de nombreux biais, on ne peut plus étudier correctement l'association causale entre un facteur d'exposition et l'apparition d'une maladie, car les biais entrainent une distorsion de la réalité. L'effet d'un biais induit une surestimation de l'association, ou à l'inverse, une sous-estimation.

Il est habituel de regrouper les biais proprement dits en trois grandes catégories qu'il est important de prendre en compte, maitriser et contenir dans des limites acceptables (cf. tableau 1).

\section{Mise en place d'une étude pharmaco- épidémiologique}

Avant qu'une étude puisse être entreprise, un important travail de préparation est nécessaire. Les détails administratifs et organisationnels doivent précéder le travail sur le terrain.

\section{Revue de la littérature et formulation d'objectifs}

La première étape de la conception d'uneétudepharmacoépidémiologique consiste à avoir une «idée » de l'étude à réaliser. Il s'agit d'une idée créatrice libre qui germe généralement de l'expérience professionnelle ou du besoin de répondre à une question particulière. Lorsqu'on a une idée sur la nature de l'étude que l'on souhaite entreprendre, il faut déterminer l'état des connaissances à ce sujet et s'assurer, grâce à une bibliographie critique, que le problème n'a pas déjà été abordé et résolu $[69,70]$.

\section{Définition de la population étudiée et calcul de la taille d'échantillon}

Il est essentiel de délimiter avec précision la collectivité où est réalisée l'étude : cette collectivité s'appellera la population cible. L'échantillonnage est réalisé le plus souvent par tirage au sort, ce qui garantit que tout sujet appartenant à la population peut faire partie de l'échantillon avec une probabilité connue [71-75].

\section{Rédaction de protocole}

Le protocole est la pierre angulaire d'un projet d'étude pharmacoépidémiologique : c'est le document dans lequel le but de l'étude, la méthode, la population étudiée et les analyses prévues sont décrits. Dans cette rubrique, on trouve les méthodes d'analyse statistique utilisées ainsi que le calcul du nombre de sujets nécessaires [69,76-81]. Les questions administratives et juridiques, les difficultés possibles et les limites, les délais de réalisation, les modalités de communication des résultats sont aussi abordés dans le protocole. Il faut écrire et suivre un bon protocole qui doit refléter les principes éthiques et légaux de la pharmacoépidémiologie. 


\begin{tabular}{|c|c|c|}
\hline Type de biais & Causes & Correction du biais \\
\hline $\begin{array}{l}\text { Biais de sélection ou } \\
\text { biais d'échantillonnage }\end{array}$ & $\begin{array}{l}\text { Échantillon non représentatif de sa } \\
\text { population-source }\end{array}$ & $\begin{array}{l}\text { - Échantillonnage aléatoire des cas et des témoins } \\
\text { - Appariement des groupes étudiés } \\
\text { - Stratification de l'échantillon de population } \\
\text { - Réduction du nombre de non-répondants et perdus } \\
\text { de vue }\end{array}$ \\
\hline $\begin{array}{l}\text { Biais d'information ou } \\
\text { de mesure }\end{array}$ & $\begin{array}{l}\text { Distorsion dans la façon dont } \\
\text { l'information concernant le paramètre } \\
\text { mesuré est recueillie selon les groupes } \\
\text { comparés. Cette distorsion est causée } \\
\text { par: } \\
\text { - une erreur de classement non- } \\
\text { différentielle; } \\
\text { - une erreur de classement différentielle. }\end{array}$ & $\begin{array}{l}\text { - Sélection dans les groupes comparés de personnes } \\
\text { aptes à répondre à des questions, recueillir des faits } \\
\text { objectifs } \\
\text { - Réalisation des mesures « en aveugle» } \\
\text { - Appui des réponses fournies par le sujet par des } \\
\text { données venant d'autres sources (dossiers médicaux, etc.) } \\
\text { - Standardisation de toutes les mesures effectuées : } \\
\text { utilisation de questionnaires validés, formation des } \\
\text { enquêteurs à l'interrogatoire, etc. }\end{array}$ \\
\hline Biais de confusion & $\begin{array}{l}\text { Défaut de prise en compte d'un facteur } \\
\text { de confusion. Il peut être également } \\
\text { causé par d'autres cas particuliers de } \\
\text { biais de confusion qui sont: } \\
\text { - le biais d'indication; } \\
\text { - le biais protopathique. }\end{array}$ & $\begin{array}{l}\text { - Analyse systématique de la littérature avant le début } \\
\text { de toute étude pharmacoépidémiologique }\end{array}$ \\
\hline
\end{tabular}

\section{Financement}

Une fois le protocole de l'étude rédigé, il faut réunir les fonds nécessaires à sa réalisation. Si le coût global du projet est faible, on peut envisager de réaliser l'étude avec les ressources disponibles. Mais les enquêtes pharmacoépidémiologiques sont souventtrès coûteusesetnécessitentun financement important. On identifie alors les organismes de financement susceptibles d'être intéressés par le projet de recherche $[20,82,83]$.

\section{Réalisation de l'étude}

L'étude commence par la recherche des médecins, pharmaciens, patients et bases de données. Les données recueillies pendant l'étude ne doivent concerner que ce qui a été défini dans le protocole, et faire l'objet d'un contrôle de la qualité qui doit être mis en œuvre dans les plus brefs délais possibles, de manière précise et intelligible $[70,84,85]$.

\section{Gestion des données}

Cette étape correspond à l'analyse des données quiconsisteàéditer les tableaux et à calculer les paramètres statistiques prévus à la phase de planification.

\section{Communication et publication des résultats}

Les résultats d'une étude pharmacoépidémiologique doivent être valorisés et mis à la disposition de la communauté scientifique sous forme de publications. De même des communications orales dans des congrès scientifiques ou des manifestations professionnelles seront envisagées [69].

\section{Conclusion}

Aujourd'hui, les études pharmacoépidémiologiques apportent des données indispensablessurlaprescriptionmédicale courante et permettent d'approcher, en situation réelle, une estimation valable du rapport bénéfice/risque d'un traitement médicamenteux permettant ainsi sa réévaluation. Cependant, cette discipline est confrontée à plusieurs défis, à savoir les possibilités de financement limitées, les restrictions réglementaires, les préoccupations de confidentialité, les possibilités de formation limitées et l'insuffisance des ressources en personnel.

Relever ces défis est l'affaire de tous les secteurs de santé publique, y compris les industries et les gouvernements. Le rapprochement entre ces différents partenaires permet d'appuyer et de soutenir le développement continu d' études pharmacoépidémiologiques d'une grande rigueur scientifique afin de maximiser le bénéfice et minimiser le risque inhérent à tous les médicaments.

Les risques de conflits d'intérêts, dans la mesure où ces études peuvent être méthodologiquement controversées et susceptibles d'avoir un fort impact sur l'économie des laboratoires, sont enfin un enjeu à prendre en compte.

\section{Références}

1. Montastruc JL. Les cinq "trop" des essais cliniques : plaidoyer pour une pharmacoépidémiologie pour tous [The five "too's" of clinical trials: a plea for pharmacoepidemiology for all]. Prescrire, 2006, 26(273):471. 
2. Bruno F. Pharmaco-épidémiologie : les enseignements de la «vraie vie» [Pharmacoepidemiology: lessons from real life]. Revue du rhumatisme, 2004, 71(5):333-336.

3. Bégaud B. Dictionnaire de Pharmaco-épidémiologie. $3^{\text {e }}$ édition [Dictionary of pharmacoepidemiology, 3rd ed.]. Bordeaux, ARME-Pharmacovigilance Éditions (Association pour la Recherche MEthodologique en Pharmacovigilance), 1998.

4. Samy S. Apport des études pharmacoépidémiologiques dans l'évaluation du risque thérapeutique [Contribution of pharmacoepidemiological studies to evaluations of treatment-related adverse events]. Revue du rhumatisme, 2006, 73(10-11):1018-1020.

5. Lawson DH. Pharmacoepidemiology: a new discipline. British Medical Journal, 1984, 289:940-941.

6. Combe B. Le thalidomide : vers de nouvelles indications [Thalidomide: new indications] ? Revue du rhumatisme, 2001, 68(10-11):951-957.

7. Amour M-C et al. Amélioration du pronostic obstétrical des femmes porteuses d'un utérus distilbène par une prise en charge adaptée [Obstetrical prognosis improvement in patients exposed in utero to diethylstilbestrol with specific care management]. Gynécologie Obstétrique \& Fertilité, 2004, 32(11):942-949.

8. Veurink M, Koster M, Berg LT. The history of DES, lessons to be learned. Pharmacy World \& Science, 2005, 27:139-143

9. Daniels SR, Hudson JI, Horwitz RI. Epidemiology of potential association between L-tryptophane ingestion and eosinophilia-myalgia syndrome. Journal of Clinical Epidemiology, 1995, 48:1413-1427.

10. Abenhaim L et al. Appetite-suppressant drugs and the risk of primary pulmonary hypertension. New England Journal of Medicine, 1996, 335:609-916.

11. Vergely $\mathrm{N}$ et al. Rhabdomyolyse métabolique au cours d'un traitement par statine [Metabolic rhabdomyolysis during statin therapy]. La revue de médecine interne, 2009, 30(8):711-713.

12. Berenbaum F. VIOXX et complications cardiovasculaires : une affaire de famille [VIOXX and cardiovascular events: a class effect ?]. Revue du rhumatisme, 2005, 72(1):1-3.

13. Bombardier $\mathrm{C}$ et al. Comparison of upper gastrointestinal toxicity of rofecoxib and naproxen in patients with rheumatoid arthritis. New England Journal of Medicine, 2000, 343:1520-1528.

14. Krumholz HM et al. What have we learnt from Vioxx? BMJ (Clinical Research Ed.), 2007, 334:120-123.

15. Bensadon AC, Marie E, Morelle A. Enquête sur le Mediator. Rapport définitif. [Mediator"survey. Final report]. Paris, Inspection Générale des Affaires Sociales, 2011 (http://lesrapports. ladocumentationfrancaise.fr/BRP/114000028/0000.pdf, consulté le 25 octobre 2011).

16. Vray M, Szafir D, Jaillon P. Pharmacoépidémiologie : identification des besoins, bases de données, critères de qualité des études [Pharmaco-epidemiology: identification of needs, databases, and study quality criteria]. Thérapie, 2001, 56:349-353.

17. Ferry S. L'usage du médicament [The use of medication]. Paris, Tec \& Doc - Éditions Médicales Internationales (EM Inter), Lavoisier, 2000.

18. Crochard-Lacour A, Lelorier J. Introduction à la pharmacoéconomie [Introduction to pharmacoeconomics]. Montréal, Les Presses de I'Université de Montréal (PUM), 2000:12.

19. Béresniak A, Bouvenot G. Utilisation de la pharmacoéconomie en antibiothérapie [Use of pharmacoeconomics in antibiotic treatment]. Médecine et maladies infectieuses, 2000, 30(3):133-140

20. Académie des Sciences (coord. A-J Valleron). L'épidémiologie humaine - conditions de son développement en France, et rôle des mathématiques [Human epidemiology - conditions for its development in France, and the role of mathematics]. Les Ulis, Éditions EDP Sciences, 2006 (Rapport sur la science et la technologie $n^{\circ} 23$ ).

21. Fresson J et al. La protection des données de santé en épidémiologie [Health data protection in epidemiology]. Revue d'Épidémiologie et de Santé Publique, 2010, 58(S1):12.

22. Vulliet-Tavernier S. La cnil et la protection des données médicales nominatives [The CNIL and the protection of personal medical data]. Médecine \& Droit, 1996, 20:2-5.

23. Cherin P. La Comission nationale de l'informatique et des libertés (CNIL). La revue de médecine interne, 1997, 18(7):512.

24. Lechopier N. Recherche et non-recherche. Les valeurs à l'œuvre dans l'évaluation des protocoles épidémiologiques [Research and non-research. The values in the evaluation of epidemiological protocols]. Revue d'Épidémiologie et de Santé Publique, 2010, 58(1):41-48.

25. Claudot F et al. Recherche en épidémiologie clinique: quelles règles appliquer [Research in clinical epidemiology: which rules should be applied ?] Revue d'Épidémiologie et de Santé Publique, 2008, 56(1):63-70.

26. Claudot F, Alla F, Essevaz-Roulet $M$. Aspects éthiques et réglementaires de la recherche en épidémiologie clinique. Guide pratique [Ethical and regulatory aspects of research in clinical epidemiology. Practical guidelines]. Revue d'Épidémiologie et de Santé Publique, 2004, 52(3):297-312.

27. Nass SJ, Levit LA, Gostin LO, eds. Beyond the HIPAA Privacy Rule: Enhancing Privacy, Improving Health Through Research. Washington DC, The National Acadamies Press, 2009.

28. Martin K et al. Où trouver des données pour la pharmacoépidémiologie en France? Quelques idées [Where can we find French data for pharmacoepidemiology? A few thoughts]. Revue d'Épidémiologie et de Santé Publique, 2007, 55(1):58-63.

29. Czernichow P, Chaperon J, Le Coutour X. Épidémiologie [Epidemiology]. Paris, Masson, 2001 (Collection Abrégés connaissances et pratique).

30. Larrousse N, Innocenti E. Création de bases de données [Creating databases]. Paris, Pearson Education France, Collection Synthex informatique, 2006:2.

31. Moszer I. Bases de données génomiques microbiennes : principes, méthodes et applications. [Microbial genomic database: principles, methods and applications]. Annales de l'Institut Pasteur / Actualités, 2002, 11:85-103.

32. Mouillet E. La recherche bibliographique en médecine et santé publique : guide d'accès [Bibliographic searching in medicine and public health: access guide]. Paris, Elsevier, 2005:43.

33. Dufour J-C, Mancini J, Fieschi M. Recherche de données factuelles [Searching for evidence-based data]. Journal de Chirurgie, 2009, 146(4):355-367.

34. Péguiron F. L'intelligence économique au service des acteurs de l'université : la question du partage de l'information sur les campus [Economic intelligence to serve university actors: the issue of information sharing on campus]. Paris, L'Harmattan, Collection Intelligence économique, 2008:61.

35. Eveillard P, Hannedouche T. Recherche bibliographique médicale avec Medline-Pubmed. Une approche pratique basée sur l'exemple [Bibliographic medical research using MEDLINE-PubMed. A practical approach based on example]. Néphrologie \& Thérapeutique, 2007, 3(7):475-485.

36. Hennessy S. Use of health care databases in pharmacoepidemiology. Basic \& Clinical Pharmacology \& Toxicology, 2006, 98:311-313.

37. North Atlantic Treaty Organization, Scientific Affairs Division. Pharmacoepidemiology studies using large databases. In: Strom BL, Velo GP, eds. Drug epidemiology and post marketing surveillance. New York, Springer, 1992:68. 
38. Bousquet PJ et al. Observatoires de santé, observatoires du médicament, vigilance et registres [Health monitoring, drug monitoring, vigilance and registers]. Revue Française d'Allergologie et d'Immunologie Clinique, 2004, 44(8):659-663.

39. Golberg $M$ et coll. L'épidémiologie sans peine. $2^{\mathrm{e}}$ édition [Epidemiology without pain, 2nd ed]. Paris, Éditions Frison-Roche, 1990.

40. Malek K. Santé publique. Médecine légale, Médecine du Travail [Public health. Forensic medicine, occupational medicine]. Paris, Éditions ESTEM, Collection Med-line, 1996.

41. Czernichow P. Santé et environnement - Maladies transmissibles [Health and environment - Communicable diseases]. Paris, Elsevier Masson, 2006.

42. Sabbah L et coll. Module 7 : Santé et environnement - Maladies transmissibles. [Module 7: Health and environment - Communicable diseases]. Paris, Éditions ESTEM, Collection DCEM, 2004.

43. Dodge Y. Premiers pas en statistique, $2^{\mathrm{e}}$ édition [First steps in statistics, 2nd ed.]. Paris, Springer-Verlag France, 2006.

44. Py B. La statistique sans formule mathématique [Statistics without mathematical formulas]. Paris, Pearson Education France, 2007:25.

45. Ardilly P. Les techniques de sondage, $2^{\mathrm{e}}$ édition [Sampling techniques, 2nd ed.]. Paris, Éditions TECHNIP, 2006.

46. Laurent C, Etard J-F. Le sondage en grappes : implications de la méthode d'analyse sur les conclusions de l'enquête [Cluster sampling: consequences of data analysis on drawing conclusions]. Revue d'Épidémiologie et de Santé Publique, 2005, 53(1):43-50.

47. Institut national d'études démographiques (INED). Démographie : analyse et synthèse - III. Les déterminants de la mortalité [Demography: analysis and synthesis - III. Determinants of mortality]. Paris, INED, 2002:29.

48. Guillemin F. Incidence et prévalence : deux indicateurs exigeants [Incidence and prevalence: two demanding indicators]. Revue du rhumatisme, 2005, 72(1):7-9.

49. Catalina P. Médecine et risque au travail: Guide du médecin en milieu de travail, $2^{\mathrm{e}}$ édition [Medicine and workplace hazard: a medical guide in the work environment, 2nd ed.]. Paris, Elsevier Masson, 2009.

50. Blanchot-Isola C. L'étude de cohorte ou de suivi [The cohort study or follow- up study]. La revue Sage-femme, 2008, 7(5):276-279

51. ARME-P. Études de cohortes en pharmacovigilance, Seconde édition [Cohort studies in pharmacovigilance, 2nd ed.]. Bordeaux, ARME-Pharmacovigilance Éditions (Association pour la Recherche MEthodologique en Pharmacovigilance), 1995.

52. Bastuji-Garin S. Notions d'épidémiologie utiles en dermatologie [Epidemiologic considerations in dermatology]. EMC - Dermatologie-Cosmétologie, 2005, 2(3):119-131.

53. Jolly D et al. Lecture critique d'articles médicaux [Critical reading of medical articles]. Paris, Elsevier Masson, Collection DCEM, 2009:4-5.

54. Gordis L. Epidemiology, 4th ed. Elsevier Health Sciences, 2009:204.

55. Ollivier $\mathrm{L}$ et al. Les mesures d'associations en épidémiologie [Correlation measures for epidemiological studies]. Médecine tropicale, 2003, 63(1):75-78.

56. Ollivier $\mathrm{L}$ et al. Les mesures d'impact en épidémiologie [Impact measures in epidemiology]. Médecine tropicale, 2004, 64(1):71-74.

57. Bousquet PJ et al. Les principales études épidémiologiques d'observation [Principal types of observational epidemiological studies]. Revue Française d'Allergologie et d'Immunologie Clinique, 2004, 44(6):509-515.
58. Salmi L-R. Lecture critique et communication médicale scientifique : comment lire, présenter, rédiger et publier une étude clinique ou épidémiologique [Critical and scientific medical communication: how to read, conduct, write and publish a clinical or epidemiological study]. Paris, Elsevier Masson, 2002.

59. Petrie A, Sabin C. Medical statistics at a glance, 2 nd ed. West Sussex, UK, Wiley-Blackwell, 2005:40.

60. Morabia A. Relative risk and odd ratio. Revue des Maladies Respiratoires, 2003, 20:757-759.

61. Clavel J, Lacour B. Méthodes épidémiologiques [Epidemiological methods]. In: Sommelat D, Clavel J, Lacour B. Epidémiologie des cancers de l'enfant [Epidemiology of childhood cancers]. Paris, Springer-Verlag, 2009:130.

62. Duhaut $\mathrm{P}$ et al. Les grands types d'études cliniques: 1 . de la descrition à l'analyse [Grand types of clinical studies : 1.from description to analysis]. La revue de médecine interne, 2006, 27(2):137-139.

63. Pearce $\mathrm{N}$. The use of beta agonists and the risk of death and near death from asthma. Journal of Clinical Epidemiology, 2009, 62:582-587.

64. Bonita R, Beaglehole R, Kjellström T. Basic epidemiology, 2nd ed. Geneva, World Health Organization, 2006:41.

65. Suissa S. Novel approaches to pharmacoepidemiology study design and statistical analysis. In: Strom BL, Kimmel SE, eds. Textbook of pharmacoepidemiology. West Sussex, UK, John Wiley and Sons, 2006.

66. Merrill RM. Introduction to epidemiology, 5th ed. Sudbury MA., Jones and Bartlett Learning, 2009:195.

67. Salas M, Stricker B. Research methods for pharmacoepidemiology studies. In: Glasser S, ed. Essentials of clinical research. Munich, Springer, 2008.

68. Viboud $\mathrm{C}$ et al. Comparison of the statistical efficiency of case-crossover and case-control designs: application to severe cutaneous adverse reactions. Journal of Clinical Epidemiology, 2001, 54:1218-1227.

69. Bourgkard E, Demange V, Aubry C. L'épidémiologie en santé au travail (II) : étapes d'une étude épidémiologique en milieu professionnel [Epidemiology in occupational health (II): steps in an epidemiological study in the workplace]. Documents pour le médecin du travail, 2008, 113:7-19.

70. Descatha $\mathrm{A}$ et al. Enquêtes épidémiologiques en milieu de travail: quelques bases pour le médecin du travail [Epidemiological survey in work environment : a few bases for the occupational physician]. Archives des maladies professionnelles et de l'environnement, 2005, 66(4):343-351.

71. Bégaud B et al. Taille d'échantillon nécessaire pour mettre en évidence une différence entre deux risques [Sample size required to demonstrate a difference between two risks]. In: Études de cohortes en pharmacovigilance, Seconde édition [Cohort studies in pharmacovigilance, 2nd ed.]. Bordeaux, ARMEPharmacovigilance Éditions (Association pour la Recherche MEtholologique en Pharmacovigilance), 1995.

72. Riou $B$, Landais $P$. Principes des tests d'hypothèse en statistique : $\alpha, \beta$ et $P$ [Principles of hypothesis testing in statistics: $\alpha$, $\beta$ et P]. Annales Françaises d'Anesthésie et de Réanimation, 1998, 17:1168-1180.

73. Strom Brian L. Sample size considerations for pharmacoepidemiology studies. In: Strom BL, Kimmel SE, eds. Textbook of pharmacoepidemiology. West Sussex, UK, John Wiley and Sons, 2006.

74. Strom BL. The promise of pharmacoepidemiology. Annual Review of Pharmacology and Toxicology, 1987, 27:71-86.

75. Tubert-Bitter $\mathbf{P}$ et al. Sample size calculations for risk equivalence testing in pharmacoepidemiology. Journal of Clinical Epidemiology, 2000, 53:1268-1274. 
76. Schmidely $\mathrm{N}$ et al. Impact du process de recrutement dans une étude pharmacoépidémiologique sur la constitution de l'échantillon de médecins [Effect of the recruitment process in a pharmacoepidemiological study on the composition of the sample of doctors]. Revue d'Épidémiologie et de Santé Publique, 2007, 55(1S):16-17.

77. Nguyen L et al. Validation d'un questionnaire de connaissances sur l'asthme [Validation of an asthma knowledge questionnaire]. Revue des Maladies Respiratoires, 2003, 20(6C1):871-880.

78. Gregg MB. Field epidemiology, 2nd ed. Oxford, Oxford University Press, 2002.

79. Russel AJ, Burnay N, Servais O. Méthodes de recherche en sciences humaines [Research methods in social and behavioural sciences]. Bruxelles, De Boeck Université, 1999:189.

80. Talley NJ, Locke GR, Saito YA, eds. Gl epidemiology. Malden, MA, Wiley-Blackwell, 2007.

81. Kauffman $\mathrm{F}$ et al. Construction et validation d'un questionnaire en épidémiologie respiratoire [Construction and validation of a respiratory epidemiological questionnaire]. Revue des Maladies Respiratoires, 2002, 19(3):323-333.
82. Dos Santos Silva I. Épidémiologie du cancer : principes et méthodes [Cancer epidemiology: principles and methods]. Lyon, Centre international de Recherche sur le Cancer, 1999.

83. Goldberg M, Zins M, Lert F. Proposition pour la création d'une plate-forme scientifique et technique pluri-organismes pour l'aide à la gestion de cohortes et de grandes enquêtes épidémiologiques. Le projet Plastico [Proposal to create a scientific platform and multi-agency technical assistance for the management of large cohort and epidemiological surveys. The Plastico project]. Institut national de la santé et de la recherche médicale (INSERM), 2007 (http://www.rppc.fr/PLASTICO-IRESP.pdf, consulté le 17 octobre 2011).

84. Papoz L. Qualité des données dans les études épidémiologiques [Quality of data in epidemiological studies]. Gérontologie et Société, 2001, 99:25-36.

85. Altpeter E, Burnaud B, Capkun G. Bonnes pratiques en épidémiologie : recommandations fondamentales [Good practices in epidemiology: basic recommendations]. Sozial- und Praventivmedizin, 2005, 50:20-23.

\section{CD-ROM International Pharmacopoeia. Fourth Edition, 2011}

This CD-ROM incorporates all new monographs, amendments and additions as adopted by the Expert Committee on Specifications for Pharmaceutical Preparations and is being published to include the First and Second Supplements of the Fourth Edition of The International Pharmacopoeia. Only the electronic version is being produced this time. Thus it will replace all former versions in a user-friendly way.

Further information about this and other WHO publications is available at: http://www.who.int/publications/en/ 